Insgesamt ist demzufolge in Polen bis 2010 mit einer durch den Beitritt bedingten Investitionsnachfrage im Umweltbereich von über 50 Milliarden DM zu rechnen (vgl. Tabelle 1). 45 Milliarden DM dürften eine Untergrenze darstellen, während die Obergrenze schwerer bestimmbar ist. Das Investitionsvolumen teilt sich zu je über 40 Prozent auf die Bereiche Wasser und Luft auf. Auf deutlich höhere Werte vom ca. 80 Milliarden DM bis 2015 kommt ein Szenario der Weltbank, das allerdings auf anderen Annahmen hinsichtlich der erforderlichen Anpassungen beruht.

Bei den Zahlenangaben zu berücksichtigen ist einerseits, dass viele Bereiche des acquis noch gar nicht erfasst werden. Andererseits ist die Annahme, dass das gesamte Potenzial bis 2010 realisierbar ist, eher optimistisch. Hier ist vor allem das Finanzierungsproblem zu nennen. Dies könnte dazu führen, dass selbst bei nominell kurzen Übergangsregelungen die praktische Implementierung 2010 noch deutlich zurückhängt.

Der Beitritt Polens zur EU erweist sich somit als komplexer Prozess. Umweltschutz ist eines der harten Themen der Beitrittsverhandlungen, da die bestehenden Unterschiede hier groß sind und die Anpassung kostspielig sein wird. $\mathrm{Zu}$ gleich wird hierdurch in Polen eine stark wachsende Nachfrage nach Umweltschutzgütern entstehen, die für Unternehmen in EU-Ländern, eine Exportchance darstellt.

\section{Anmerkungen}

(1) Vgl. hierzu auch Nill, Jan/ Petschow, Ulrich: Die vernachlässigte Dimension der EU-Osterweiterung. In: Ökologisches Wirtschaften Nr. 1/1999, S. 5-6.

(2) Für eine ausführliche Darstellung des folgenden vgl. Nill, Jan/ Lünenbürger, Benjamin/ Petschow, Ulrich: Ein Szenario der Rolle des Umweltschutzes beim EU-Beitritt Polens. Schriftenreihe des IÖW 154/00, Berlin 2000.

(3) Vgl. Jedrysik, Eugeniusz: Der Nationale Fond für Umweltschutz als Finanzierungsinstrument der Umweltpolitik Polens. In: Europäische Akademie für städtische Umwelt Berlin (Hrsg.): Infrastruktur im Wandel. Ergebnisse einer Tagung. EA.UE Berlin 1999, S. 23-32.

(4) Vgl. auch Homeyer, Ingmar von/ Klasing, Anneke/ Kempmann, Lena: EU Enlargement: Screening Results in the Environmental Sector. In: Environmental Law International Newsletter Nr. 2/1999, S. 43-47.
(5) Das Szenario ist ein Baustein des Projekts "Markterkundung im Umweltbereich für kleine und mittlere Unternehmen aus Brandenburg in Polen", das unter Leitung von Prof. Dr. Stefan Zundel von der Fachhochschule Lausitz 1999 bis 2000 im Rahmen des BMBF-Programms Anwendungsbezogene Forschung an Fachhochschulen durchgeführt wurde. Für eine ausführliche Darstellung von Annahmen, Methoden und Detailergebnissen vgl. Nill/Lünenburger/ Petschow (siehe Anm. 2).

(6) Vgl. Jantzen, Jochem: Cost of Compliance with EU environmental directives in Poland, paper for seminar 10.3.99 at the Polish Ministry for Environmental Protection, TME 1999; Hughes, Gordon/ Bucknall, Julia: Poland, Complying With EU Environmental Legislation. ECSSD Working Paper No. 14, The World Bank, Washington 1999.

\section{Die Autoren}

Jan Nill und Ulrich Petschow sind wissenschaftliche Mitarbeiter am IÖW. Benjamin Lünenbürger studiert Volkswirtschaft an der TU Berlin.

Kontakt: IÖW, Potsdamer Str. 105, 10785 Berlin.

Tel. 030/ 884594-0, Fax 030/ 8825439, E-mail:

Jan.Nill@ioew.de, Ulrich.Petschow@ioew.de

Umweltorientierte Studieninhalte in den Wirtschaftswissenschaften an deutschen Hochschulen

\title{
Integration auf dem Weg
}

\section{Für die Umsetzung des Konzepts einer nachhaltigen Entwicklung tragen die Hochschulen eine be- sondere Verantwortung. Hier werden zukünftige Führungskräfte und damit Multiplikatoren aus- gebildet. Eine umfassende empirische Analyse zeigt, dass in den Wirtschaftswissenschaften um- weltorientierte Studieninhalte zunehmende Verbreitung gefunden haben. Dennoch bleiben Defizi- te bestehen.}

D Grenzen des Wachstums wird an deutschen Hochschulen schon lange geführt. Als Lösungspfad wurde das Konzept des Sustainable Development aufgezeigt. Dessen Umsetzung erfordert ein tiefgreifendes Umdenken in vielen Bereichen des menschlichen Handelns. Dies setzt, wie psychologische, pädagogische und umweltpolitische Forschungsarbeiten zeigen, Umweltwissen und Umweltbewusstsein voraus. Deshalb kommt der Umweltbildung, speziell auch der Lehre und Forschung an Universitäten und Hochschulen eine besondere Bedeutung $\mathrm{zu}$. Hier werden zukünftige Führungskräfte ausgebildet, denen besondere Aufmerksamkeit geschenkt werden muss, weil sie zukünftig als gesellschaftliche Multiplikatoren für einen verantwortungsvollen Umgang mit der Natur wirken.

Dies spiegelt sich auch in der im Rahmen der Agenda 21-Diskussion entwickelten COPERNICUS-Charta wider. Diese von vielen europäischen Universitäten unterzeichnete Absichtserklärung beinhaltet unter anderem ein Aktionsprogramm, welches insbesondere die inhaltliche Anreicherung der Lehre und Forschung um ökologische Aspekte anspricht (1).

Inwieweit sich dies auch in den Lehrveranstaltungen wiederfindet, wurde in einer Analyse der Umweltorientierung der Wirtschaftswissenschaften an deutschen Hochschulen erhoben (2). Dazu wurden umweltorientierte Lehrprogramme von 43 Universitäten, 3 Gesamthoch- schulen und 19 Fachhochschulen hinsichtlich Art und Umfang der Integration umweltorientierter Studieninhalte untersucht. Erfasst wurden 89 Fächer mit Umweltbezug, welche wiederum für 104 verschiedene Studiengänge angeboten werden. Die unterschiedlichen Zahlen erklären sich dadurch, dass an einigen Hochschulen in den Wirtschaftswissenschaften mehrere Fächer mit Umweltbezug gelehrt werden, welche zum Teil wiederum mehreren Studiengängen offen stehen.

Somit können, obwohl die Analyse nicht auf einer Vollerhebung beruht, aufgrund der Vielzahl der untersuchten Hochschulen doch Tendenzaussagen getroffen werden. Diese betreffen

- die Art der Studiengänge, in die umweltorientierte Aspekte integriert wurden,

- die organisatorische Verankerung der entsprechenden Fächer im Studium sowie

- die konkreten Inhalte und darauf aufbauend Potenziale und Probleme.

\section{Vielfältige Formen und Inhalte}

Eine ökologieorientierte Ausbildung im Wirtschaftsbereich erfolgt in Deutschland sowohl an den Universitäten als auch an den Fachhochschulen in sehr vielfältiger Weise. Sie wurde in den verschiedensten Studiengängen, zum Beispiel Betriebs- und Volkswirtschaftslehre, Wirtschaftsin- 
genieurwesen, Wirtschaftswissenschaften, Wirtschaftsinformatik und Wirtschaftspädagogik, integriert. Die BWL bildet jedoch den Schwerpunkt. Darüber hinaus gibt es aber auch Hochschulen mit speziell ökologisch ausgerichteten Studienrichtungen:

Ökonomie mit ökologischem Schwerpunkt Carl von Ossietzky Universität Oldenburg (3);

- Umwelt- und Betriebswirtschaft, Wirtschaftsund Umweltrecht - beide Fachhochschule Trier (Birkenfeld); und

- Umweltwissenschaften - Universität Lüneburg, als interdisziplinärer Studiengang mit wirtschaftswissenschaftlichen Teilen.

Somit ist eine Minderheit von 4 Prozent der erfassten Fächer als eigener ökologieorientierter, wirtschaftswissenschaftlicher bzw. interdisziplinärer Studiengang einzuordnen. Verbreiteter sind mit 17 Prozent für alle Studierenden obligatorische Pflichtfächer sowie Wahlfächer mit 24 Prozent. Den Schwerpunkt bilden mit 55 Prozent der erfassten Fächer Spezialisierungen im Rahmen einer speziellen BWL oder eines Wahlpflichtfaches. zuordnen lässt. In weiteren 17 Prozent der Fächer erfolgt die umweltorientierte Erweiterung in klassischen BWL-Fächern wie Produktionswirtschaft oder Kostenrechnung. 19 Prozent stammen aus anderen Disziplinen wie Ingenieurswissenschaften oder dem Recht.

Grundsätzlich lässt sich deshalb feststellen, dass ein ansatzweise interdisziplinäres Vorgehen in allen untersuchten Studienangeboten anzutreffen ist. In vier Fällen setzen sich die Studienangebote direkt aus Vorlesungen verschiedener Fachbereiche zusammen.

\section{Fehlende internationale Ausrichtung}

Bei der Vielfältigkeit der betrachteten Aspekte im nationalen Kontext fällt auf, dass eine internationale Ausrichtung zu Fragestellungen der ökologieorientierten Wirtschaftswissenschaften bisher kaum erfolgt. Insbesondere hinsichtlich Mittel- und Osteuropa kann in diesem Bereich vor dem Hintergrund der zunehmenden Globalisierung der Märkte und der EU-Osterweiterung ein Defizit festgestellt werden (4).

\begin{tabular}{|c|c|c|c|}
\hline Studieninhalt & Fächerzahl & Studieninhalt & Fächerzahl \\
\hline Ökonomie/Ökologie & $70(78,7 \%)$ & Umweltmanagementinstrumente & $57(64,0 \%)$ \\
\hline Globale Umweltprobleme & $31(34,8 \%)$ & - Input-0utput-Analysen & $26(29,3 \%)$ \\
\hline Kommunale Umweltprobleme & $14(15,7 \%)$ & • Ökobilanzen & $40(44,9 \%)$ \\
\hline Umweltpolitik & $59(66,3 \%)$ & - Umweltkostenrechnung & $28(31,5 \%)$ \\
\hline Umweltrecht & $46(51,7 \%)$ & - Öko-Controlling & $41(46,1 \%)$ \\
\hline Strategisches Umweltmanagement & $48(53,9 \%)$ & - Öko-Audit & $39(43,8 \%)$ \\
\hline Operatives Umweltmanagement & $68(76,4 \%)$ & - Öko-Labeling & $15(16,9 \%)$ \\
\hline \multirow{2}{*}{\multicolumn{2}{|c|}{$\begin{array}{l}\text { insb. in den Bereichen Produktion (39) und Marketing (33), } \\
\text { am wenigsten in Finanzierung (11) und Personalwirtschaft (19) }\end{array}$}} & Umwelttechnik & $34(38,2 \%)$ \\
\hline & & Umweltpsychologie & $25(28,1 \%)$ \\
\hline Umweltinformatik & $22(24,7 \%)$ & \multirow{2}{*}{$\begin{array}{l}\text { Internationale Ausrichtung } \\
\text { - davon Osteuropa }\end{array}$} & $25(28,1 \%)$ \\
\hline Umweltmanagementsysteme & $44(49,4 \%)$ & & $10(11,2 \%)$ \\
\hline
\end{tabular}

Analog zur Vielzahl umweltorientierter Studienarten verwundert das reichhaltige Angebot an Studieninhalten nicht sonderlich (vgl. Tab. 1). Es deckt alle überprüften Aspekte ab. Besonders häufig werden in den erfassten Fächern der Zusammenhang Ökonomie/ Ökologie, operatives Umweltmanagement, Umweltpolitik sowie Umweltmanagementinstrumente betrachtet, während Randgebiete wie Umweltinformatik und Umweltpsychologie nur begrenzt Beachtung finden.

Deutlich wird dies auch in der vorgenommenen Klassifizierung der Fächer nach inhaltlichen Schwerpunkten. Sie zeigt, dass sich die Mehrzahl der Fächer ökologieorientierter spezieller BWL (36 Prozent), ökologieorientierter spezieller VWL (21 Prozent) sowie BWL- und VWLübergreifender Spezialisierungen (7 Prozent)
Deshalb wurde am Internationalen Hochschulinstitut Zittau im Rahmen einer Förderung durch die Deutsche Bundesstiftung Umwelt die spezielle BWL Internationales und interdisziplinäres Umweltmanagement aufgebaut (5), deren Curriculum Umweltpolitik, Umweltrecht, Umweltmanagement und Umwelttechnik im deutsch-polnischtschechischen Vergleich darstellt und somit in polnische und tschechische Universitäten transferierbar ist (6).

Zusammenfassend lässt sich feststellen, dass eine recht breite Umweltorientierung in den Wirtschaftswissenschaften erfolgt, allerdings auf unterschiedlichem Niveau. Notwendig ist sicher eine Verankerung von Umweltaspekten in Pflichtveranstaltungen, um zukünftigen Wirtschaftswissenschaftlern Einblicke in die zahlreichen Wechsel-

wirkungen von Ökonomie und Ökologie sowie daraus erwachsenden Entwicklungen der Gesellschaft zu geben. Die Tendenz scheint dabei, den Anforderungen des Arbeitsmarktes entsprechend, eher zu einer Integration von Umweltaspekten in „klassische“ BWL-Fächer als zu einer Etablierung neuer Spezialisierungen zu gehen, wobei internationale Ansätze zukünftig wohl an Bedeutung gewinnen werden.

\section{Anmerkungen}

(1) Vgl. hierzu auch das Spezial Greening the Universities in Ökologisches Wirtschaften Nr. 3-4/2000, insbesondere die Beiträge von Michelsen/ Schwiersch und Volkens/ Klaffke.

(2) Erfassungszeitraum von SS 98 bis WS 99/00; Datenermittlung in 2 Phasen; 1. Phase: Datensammlung auf Basis von Informationsanfragen und Internetrecherche der Hochschulen aus Raabe Fachverlag für Wissenschaftsinformation (Hrsg.): Deutscher Hochschulführer, 56., neubearbeitete Aufl., Stuttgart 1996; De Haan, Gerhard/ Donning, Irene/ Schulte, Bernhard: Der Umweltstudienführer, Stuttgart 1999; Wissenschaftliche Kommission Umweltwirtschaft des Verbandes der Hochschullehrer für Betriebswirtschaft e.V.; 2. Phase: Korrektur der erfassten Daten durch ermittelte Hochschullehrer auf Grundlage eines Fragebogens.

(3) Vgl. hierzu auch Pfriem, Reinhard: Ökologische Ökonomie und Innovationen kombinieren. In: Ökologisches Wirtschaften, Nr. 5/1997, S. 31-32.

(4) Vgl. Kramer, Matthias: Internationales Umweltmanagement in Mittel- und Osteuropa, in: Seidel, Eberhard (Hrsg.): Betriebliches Umweltmanagement im 21. Jahrhundert, Berlin u.a. 1999, S. 161-162.

(5) Nähere Informationen unter http://www.ihi-zittau.de/ bwl/projekte/iiu/index_iiu.html.

(6) Vgl. Brauweiler, Jana/ Rheinländer, Kathrin/ Urbaniec, Maria/ Sucharda, Jiri/ Kramer, Matthias: Ableitungen für Anforderungen an ein innovatives Ausbildungskonzept auf Basis der Analyse des Standes der Umweltbildung im deutsch-polnisch-tschechischen Vergleich, unveröffentlichter Abschlussbericht zur empirischen Analyse „Universitäre Umweltbildung in Deutschland, Polen und Tschechien" IHI Zittau 2000

Die Autorlnnen

Kathrin Rheinländer ist wissenschaftliche Mitarbeiterin am Lehrstuhl Betriebswirtschaftslehre von Prof. Dr. Matthias Kramer am Internationalen Hochschulinstitut Zittau.

Kontakt: IHI Zittau, Markt 23, 02763 Zittau.

Tel. 03583/ 7715-72, Fax -35,

E-mail: krheinlaender@ihi-zittau.de 
(c) 20I0 Authors; licensee IÖW and oekom verlag. This is an article distributed under the terms of the Creative Commons Attribution Non-Commercial No Derivates License (http://creativecommons.org/licenses/by-nc-nd/3.o/), which permits unrestricted use, distribution, and reproduction in any medium, provided the original work is properly cited. 